\title{
A Dynamic Evidential Fusion Network for Decision Making in Multi- Robot System
}

\author{
Hyun Lee \\ Sun Moon University, mahyun91@sunmoon.ac.kr
}

\begin{abstract}
In smart environments, a multi-robot system is difficult to achieve a high confidence level of information for reliable decision making. The pieces of sensed information obtained from a multi-robot system have different degrees of uncertainty. Generated contexts about the situation of the environment can be conflicting even if they are acquired by simultaneous operations. In particular, unpredictable temporal changes in sensory information reduce the confidence level of information then lead to wrong decision making. In order to solve this problem, we propose a reasoning method based on Dynamic Evidential Fusion Network (DEFN). First, we reduce conflicting information in multi-sensor networks using Evidential Fusion Network (EFN). Second, we improve the confidence level of information using Temporal Belief Filtering (TBF) and Normalized Weighting technique. We distinguish a sensor reading error from sensed information. Finally, we compare our approach with a fusion process based on Dynamic Bayesian Networks (DBNs) using paired observations so as to show the improvement of our proposed method.
\end{abstract}

Keywords: Dynamic Evidential Fusion Network, Temporal Belief Filtering, Normalized Weighting technique, Dynamic Bayesian Networks, Decision Making, Multi-Robot System

\section{Introduction}

In smart environments, a multi-robot system utilizes different types of sensors, actuators, RFID devices, and time stamps for remote- or self-control, environmental monitoring, localization and object tracking, surveillance, and so on [1], [2], [3], [42]. Particularly, the sensors and actuators attached on the multi-robot system are operated by pre-defined rule or learning process of the expert system [40], [41]. Based on the integration of the sensed data, the expert system can estimate or can make a decision about different situations of the environment. However, the pieces of information obtained from different sources can be imperfect due to the imperfection of information itself or unreliability of the sensed data. In order to deal with different aspects of the imperfection of information, we proposed Evidential Fusion Network (EFN) based on Dezert-Smarandache Theory (DSmT) [4], Proportional Conflict Redistribution no. 5 (PCR5) [5] rule, and Generalized Pignistic Transformation (GPT) [6] as a mathematical tool in [7].

However, it is still difficult to achieve a high confidence level of information for reliable decision making. Unpredictable temporal changes in sensory information may happen [8]. The previous work [7] did not consider dynamic metrics [9] of the context that shows information of the situation of the environment. We need a hybrid system that consists of both continuous time and discrete state with defined time stamp to make a correct decision about the situation. Thus, we propose Dynamic Evidential Fusion Network (DEFN) based on autonomous learning process so as to infer future information autonomously then to make a decision more correctly. The proposed DEFN deals with the relation dependency of consecutive time indexed states of information by applying Temporal Belief Filtering (TBF) algorithm. The defined discrete time domain shows a fusion process based on the timeindexed state and the defined discrete event domain shows an autonomous learning process based on the relation and temporal dependency between time stamp and the event. For instance, information obtained from each sensor are integrated and fused at each time-indexed state then the combination of estimated information and gathered information are processed in reward state of autonomous learning process for decision making.

In addition, we deal with both relative and individual importance of each component of a multirobot system such as walking robot, flying robot, and module-based robot so as to obtain optimal weights of each component of a multi-robot system based on the dynamic normalized weighting technique [10]. We then apply dynamic weights into DEFN to infer the situation of the environment for 
helping to decision making. Finally, we compare our approach with a fusion process based on Dynamic Bayesian Networks (DBNs) [11], which has the same assumption of the environment in order to show the improvement of our approach. The main contribution of the proposed DEFN is distinguishing the sensor reading error from new sensor activations or deactivations by considering the TBF algorithm and optimal weights of each component of a multi-robot system based on the normalized weighting technique. This advantage helps to make correct decisions about the situation of the environment in multi-robot utilized applications.

The rest of the paper is organized as follows. The previous work, Evidential Fusion Network [7], is introduced as a basic of reasoning method in section 2. In section 3, we introduce Dynamic Evidential Fusion Network as an autonomous learning method for reliable decision making. We perform a case study to show the improvement of our approach based on the applied multi-robot system applications in section 4. We compare and analyze the results of our approach with that of DBNs in section 5. Section 6 introduces some related works. We then conclude the paper in section 7.

\section{Evidential Fusion Network (EFN)}

A multi-robot system consists of sensors, actuators, RFID devices, and time stamps, is used for remote- or self-control, environmental monitoring, localization, or surveillance. In this case, robots are operated by pre-defined rules or learning processes of the expert systems. First, each sensor can be represented by an evidential form such as "active" (1) and "inactive" (0) to represent the status of robots or to operate actuators. Whenever the state of a certain context associated with a sensor attached on the robot is changed, the value of a sensor can change from 0 to 1 or from 1 to 0 . We simply express the status of each sensor as a frame: $\theta=\{1,0\}$. Sensed data are inherently unreliable or uncertain due to technical factors. Different types of a sensor may have various discounting factors (D) $(0 \leq D \leq 1)$. We express the degree of reliability, which is related in an inverse way to the discounting factor. The smaller reliability $(\mathrm{R})$ corresponds to a larger discounting factor $(\mathrm{D})$ (e.g., $\mathrm{R}=1-\mathrm{D}$ ).

For inferring the situation of the environment based on evidential theory, reliability discounting methods that transform belief of each source are used to reflect sensor's credibility in terms of discount factor (D). The discount mass function is defined as eq. (1). The source is absolutely reliable $(\mathrm{D}=0)$, the source is reliable with a discounting factor (D) $(0 \leq \mathrm{D} \leq 1)$, and the source is completely unreliable (D $=1)$.

$$
m^{D}(X)= \begin{cases}(1-D) m(X) & X \subset \Theta \\ D+(1-D) m(\Theta) & X=\Theta\end{cases}
$$

The basic idea of DSmT [12] is to consider all elements of $\theta$ as not precisely defined and separated.

Shafer's model [13] assumes $\theta$ to be truly exclusive and appears only as a special case of the DSm hybrid model in DSmT. Second, we use Proportional Conflict Redistribution no. 5 (PCR5) rule [5] as a conjunctive rule of combination. In DSmT framework, the PCR5 rule redistributes partial conflicting mass only to the elements involved in that partial conflict. For this approach, the PCR5 rule calculates the conjunctive rule of the belief masses of sources. It then calculates the total or partial conflicting masses. And last, it proportionally redistributes the conflicting masses to nonempty sets involved in the model according to all integrity constraints. Third, we use a disjunctive combination rule to compute prediction from previous mass distributions and model of evolution of TBF [14]. For instance, TBF, which reflects that only one hypothesis concerning activity or operation is true at each time-indexed state, ensures a temporal consistency with exclusivity. Fourth, decisions are achieved by computing the expected utilities when a decision must be taken. The maximum of the pignistic probability is used as a decision criterion in this paper. Based on Smets' notation [15], generalized pignistic transformation (GPT) is defined by [6]:

$$
P\{A\}=\sum_{X \in D^{\ominus}} \frac{C_{M}(X \cap A)}{C_{M}(X)} m(X)
$$




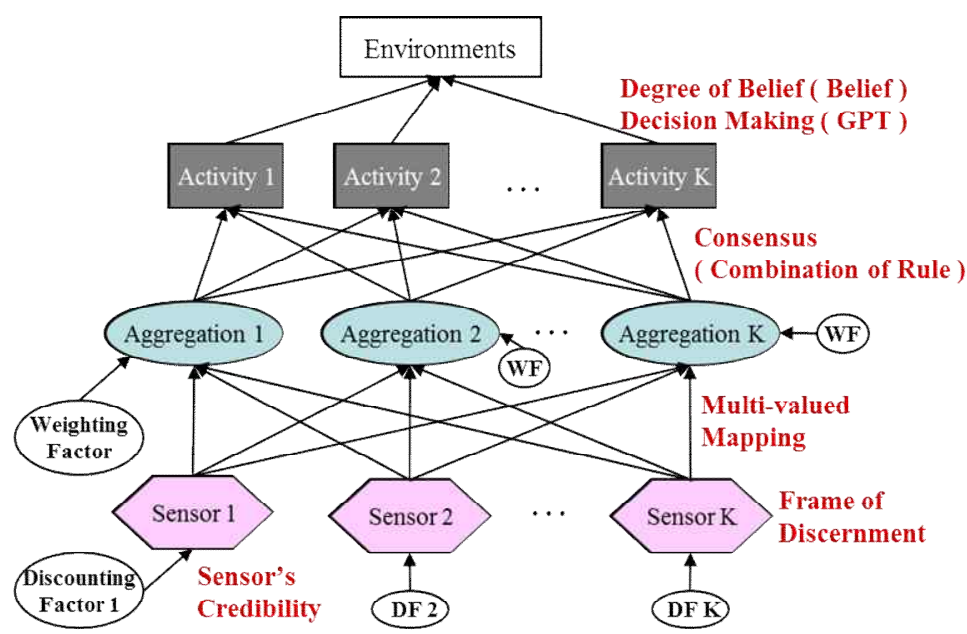

Figure 1. Evidential Fusion Network (EFN)

where $C M(X)$ denotes the DSm cardinal of a proposition $\mathrm{X}$ for the DSm model $M$ of the problem under consideration. For example, we gets a basic belief assignment with non-null masses only on $X_{1}, X_{2}$ and $X_{1} \cup \quad X_{2}$. Finally, we perform the proposed Evidential Fusion Network (EFN), shown in Figure 1, as a fusion process as follows.

1) Define the Frame of Discernment: the evidential form represents all possible sensor values and their combination values.

2) Sensor's Credibility: Reliability discounting mass functions defined as eq. (1) transforms beliefs of individual evidence in order to reflect sensor's credibility. We apply a discounting factor (D) into each sensor within EFN.

3) Multi-valued Mapping: A multi-valued mapping represents evidence to the same problem with different views. It is applied to the sensor to represent the relationships between sensors and associated objects by translating mass functions. It also can be applied to the related aggregation to represent the relationships among sensors. Each aggregation consists of different pre-defined static weights of the evidence (Relative importance).

4) Consensus: Several independent sources of evidence combine belief mass distributions on the same frame to achieve conjunctive consensus with conflict masses. We apply the PCR5 rule [5] into aggregation state to obtain a consensus that helps to recognize the situation of the environment.

5) Degree of Belief: Lower bounds (Belief (Bel)) and upper bounds (Plausibility (Pl)) on probability is calculated to represent the degree of belief. Then the uncertainty levels (P1 - Bel) of evidence is measured by using belief functions after applying the PCR5 rule.

6) Decision Making: As a decision criterion, we utilize the expected utility and the maximum of the pignistic probability such as GPT. The situation of the environment is inferred by calculating the belief, uncertainty, and confidence (i.e., GPT) levels of information.

\section{An Autonomous Learning Method}

As shown in Figure 2, Information of the environment has the association or correlation between two consecutive time-indexed states. The EFN should include a temporal dimension for dealing with this reasoning over time. Hence we propose DEFN as an autonomous learning method in this section.

\subsection{A Normalized Weighting for Evidence}

We define sensor rules to represent dynamic weights of a sensor. Within the EFN, each aggregation of sensors has the same weight. When $C\left(a_{t}^{k}\right)$ reflects the increase or decrease degree of a particular sensor, $L\left(a_{t}^{k}\right)$ reflects the level of a particular sensor. We calculate the relative weight of a sensor based on Multi-Attribute Utility Theory (MAUT) [10], [16] to setup the initial weight of a sensor within a 
given aggregation of sensors. The weights are determined by their importance in regarding to the specific situation of the environment. We construct a scale representing the properties of the levels of a sensor so as to evaluate sensors. For instance, we assume that the scale from 0 (e.g., the least affection) to 100 (e.g., the most affection) for the situation serves as measure of the evaluation as shown in Table 1. We pre-define the scale of a sensor then calculate the relative importance of a sensor. Relative weight of a sensor is equal to the sum of the value of Scale-R and Scale-E for one sensor type divided by the total sum of the value of Scale-R and Scale-E. After calculating relative weight of a sensor, we redistribute the weight of a sensor over time based on the pre-defined rule of a sensor.

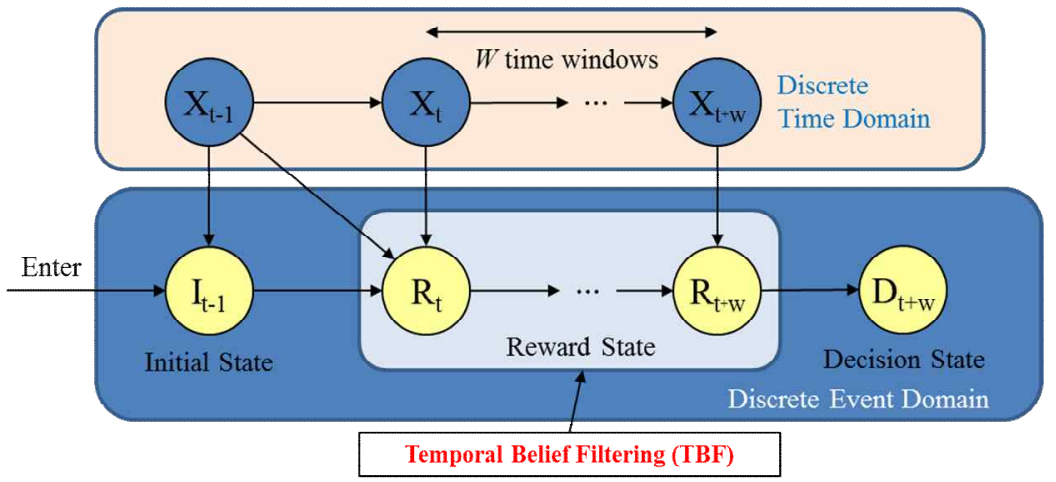

Figure 2. The relation dependency of consecutive time-indexed state with Temporal Belief Filtering

Table 1. An example of Relative Weight of a Sensor

\begin{tabular}{c|c|c|c}
\hline Sensor Type & Regular & Emergency & Relative Weight of a Sensor \\
\hline Walk-Audio & Scale-R (5) & Scale-E (55) & 0.3 \\
Walk-Light & Scale-R (5) & Scale-E (35) & 0.2 \\
Walk-Video & Scale-R (5) & Scale-E (95) & 0.5 \\
\hline Flying-Audio & Scale-R (5) & Scale-E (20) & 0.17 \\
Flying-Light & Scale-R (5) & Scale-E (45) & 0.33 \\
Flying-Video & Scale-R (5) & Scale-E (70) & 0.50 \\
\hline
\end{tabular}

Let $\mathrm{w} 1, \mathrm{w} 2, \mathrm{w} 3, \cdots, \mathrm{w} k, \cdots, \mathrm{w} k+m, \cdots, \mathrm{w} N$ denote an initial relative weight associated with a given aggregation of sensors $\left(S_{t}^{i}\right)$ for fusion process. Within same region, a normalized weighting technique for individual difference between two consecutive time-indexed states is applied to each sensor. For instance,

1) Repeat for each sensor $k$ :

$$
\begin{aligned}
& \text { if all } L\left(a_{t}^{k}\right)=L\left(a_{t+1}^{k}\right) \text { or all } C\left(a_{t+1}^{k}\right) \text { are equal, } \\
& \text { then all } \omega_{k}=\omega_{i} \\
& \text { else if any } L\left(a_{t}^{k}\right) \neq L\left(a_{t+1}^{k}\right) \text { or any } C\left(a_{t+1}^{k}\right) \text { is } \\
& \text { different, } \\
& \text { then } \hat{\omega}_{k}=\omega_{i} / \sum_{j=1}^{N}\left(\omega_{j} \pm C\left(a_{t+1}^{j}\right)\right) \text {, } \\
& \text { where } \hat{\omega}_{k} \text { defines a new weight for a sensor }
\end{aligned}
$$

\subsection{Temporal Belief Filtering (TBF)}

Depending on temporal changes, the values of a sensor at the current time-indexed state $\left(S_{t}\right)$ are evolved by the measured values at the previous time-indexed state $\left(S_{t-1}\right)$, because belief mass distribution can not vary abruptly between two consecutive time-indexed states. In order to deal with this evolution, we utilize an autonomous learning process that has three states: 1) Initial State, 2) Reward State, and 3) Final Decision State as shown in Figure 2. Particularly, Temporal Belief Filtering (TBF) operations: prediction, fusion, learning and update perform in reward state to obtain the relation-dependency. TBF ensures temporal consistency with exclusivity between two consecutive time-indexed states when only one hypothesis concerning activity is true at each time stamp. TBF 
assumes that General Basic Belief Assignment (GBBA) at current time stamp $t$ is close to GBBA at the previous time stamp $t-1$. Based on this assumption, the evolution process predicts a current GBBA taking the GBBA at $t-1$ into account. The TBF at each time stamp $t$ consists in four steps: 1) Prediction, 2) Fusion, 3) Learning and 4) Updated rule if required. For instance, if the situation of the environment was emergency (E) at $t-1$ then it would be partially emergency (E) at $t$. This is an implication rule for emergency $(\mathrm{E})$ which can be weighted by a confidence value of $m_{\mathrm{E}}\{.\} \in[0,1]$. The vector notation of a GBBA defined on the frame of discernment $(\theta)$ is used:

$$
m^{\Theta}=\left[\begin{array}{llll}
m^{\Theta}(\emptyset) & m^{\Theta}(\neg E) & m^{\Theta}(E) & m^{\Theta}(\neg E \cup E)
\end{array}\right]
$$

The evolution process can be interpreted as a GBBA defined as:

$$
m_{E}^{\Theta}=\left[\begin{array}{llll}
0 & 1-P l_{E} & B e l_{E} & P l_{E}-B e l_{E}
\end{array}\right]^{T}
$$

\subsubsection{Prediction}

We use the disjunctive combination rule $\left(M_{\cup}\right)$ to compute prediction from the previous GBBA at $t-1\left(m_{\mathrm{t}-1}\right)$ and model of evolution $\left(m^{\Theta}{ }_{M}\right)$ based on current model $M$ with only two focal sets. The disjunctive combination rule does not allow to assigning more belief to a hypothesis than does the previous GBBA. This is well suited for an autonomous evolution process under uncertainty:

$$
\hat{m}_{t, M}^{\Theta}=m_{t-1}^{\Theta}\left(M_{\cup}\right) \quad m_{M}^{\Theta}
$$

The prediction for emergency $(E)$ situation of the environment at time stamp $t$ is defined as:

$$
\hat{\mathbf{m}}_{t, E}^{\Theta}=\left[\begin{array}{c}
0 \\
\left(1-P l_{E}\right) \times m_{t-1}^{\Theta}(\neg E) \\
B e l_{E} \times m_{t-1}^{\Theta}(E) \\
1-\left[\left(\left(1-P l_{E}\right) \times m_{t-1}^{\Theta}(\neg E)\right)\right. \\
\left.+B e l_{E} \times m_{t-1}^{\Theta}(E)\right]
\end{array}\right]
$$

when $m_{\mathrm{E}}=1$, the prediction reflects a total confidence. When $m_{\mathrm{E}}=0$, the prediction reflects a total ignorance with a current time-indexed state

\subsubsection{Fusion, Learning, and Updated Rule}

Prediction and measurement represent two distinct pieces of information. Fusion of two distinct pieces of information leads to a new GBBA whose conflict value $\left(C_{F}\right)$ is relevant for belief learning and update requirement. In this case, conflict value $\left(C_{F}\right)$ is calculated by the conjunctive combination rule $\left(M_{\cap}\right)$ of prediction and measurement.

$$
C_{F}=\hat{m}_{t, M}^{\Theta}\left(M_{\cap}\right) \quad m_{t}^{\Theta}(\emptyset)
$$

Policy is required to analyze whether the current model $M$ is valid or not. If $C_{F}$ is not greater than the pre-defined threshold $(T)$, the model at $t-1$ is kept as valid at $t$. However, if $C_{F}$ exceeds $T$, the model is evolved based on the result of a conjunctive combination rule of prediction and measurement. Depending on the applied policy, the evolution process (i.e., learning) is performed.

After a learning step, a fading memory process $\left(F_{a}\right)$ has embedded to reduce relation-dependency of the pieces of long past information even though the cumulative sum of conflict value $(C F)$ between prediction and measurement is lower than $T$ during long time intervals. $F_{a}$ resets the cumulative sum of $C_{F}$ as a zero (0) and prediction is equal to measurement at time window size $(W)$, which is chosen as a constant value $(C)$. Then, updated rule is applied to the model of evolution repeatedly after $F_{a}$ is applied to measurement at time window size $(W)$. 


\subsubsection{Decision Rule}

We take the maximum of GPT (i.e., eq. (2)) as a decision criterion within the DSmT framework after the evolution process is performed. We adopt Shafer's model [13] to compare our approach with DBNs, which can get a BBA with non null masses only on $\theta_{1}$ and $\theta_{2}$ where $\theta_{1}$ and $\theta_{2}$ are hypotheses of the frame of discernment $(\Theta)$. It is required to assess the recognition performance of a time-indexed state to decide whether a temporal sequence of the state has a false alarm or new sensor activation within the defined time window size $(W)$. It is necessary to find a quality criterion without references to assess this performance.

In addition, we defined $D F$ as the differentiation of GPTs of two consecutive time-indexed states. The $D_{M}$ is defined as the mean of $D_{F}$ within the defined $W$ as the chosen criterion to distinguish a sensor reading error from new sensor activations or deactivations. If $D M$ is less than $\delta$, there is no error within $W$. If $D_{M}$ is located between $\delta$ and $\gamma$, a false alarm happens. And if $D_{M}$ is greater than $\gamma$, the emergency situation of the user or environment progress. In this case, $\delta$ is the defined false alarm threshold and is the defined emergency progress threshold for the chosen criterion. The value of $\delta$ is always lower than that of $\gamma$, because we assume that the false alarm does not often happen when new sensor (de)activation is detected by expert system in emergency situation of the environment. Based on the defined threshold $(T)$ for conflict value $\left(C_{F}\right)$ and time window size $(W)$, we can distinguish a sensor reading error from new sensor operations. We can perform evolution operations with DEFN in order to improve the confidence (i.e., GPT) level of information.

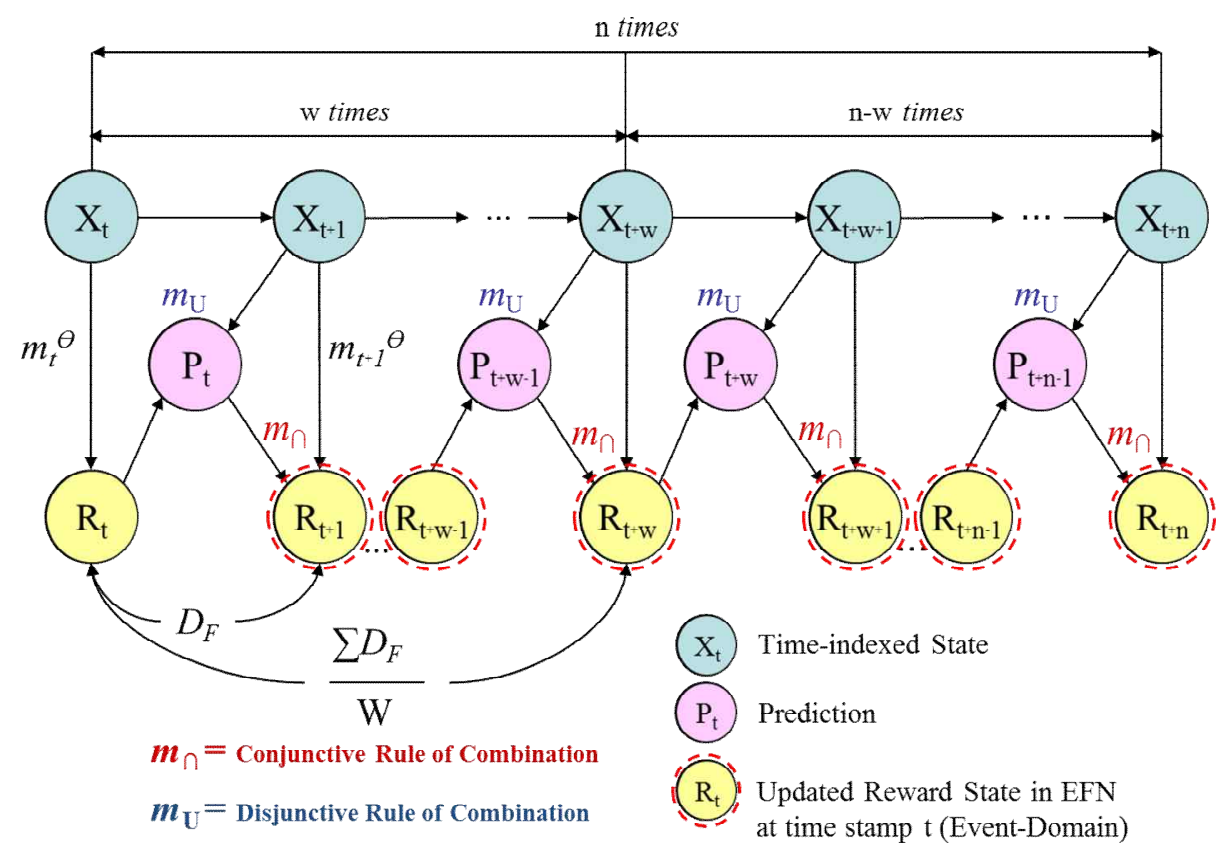

Figure 3. Dynamic Evidential Fusion Network (DEFN)

\subsection{Proposed Autonomous Learning}

Based on the EFN with a temporal dimension, the proposed DEFN is constructed as shown in Figure 3. In DEFN, a reasoning method is performed to find a false alarm in captured contexts by multi-robot and to make a high confidence level of the situation of the environment. We assume that the initial prediction is equal to the $1_{s t}$ measurement at $1_{\text {st }}$ time-indexed state $\left(S_{1}\right)$. The consecutive processing of two combination rules (i.e., disjunctive rule and conjunctive rule) is well adapted to EFN so as to update the belief mass distribution of EFN at time-indexed states. In Figure 3, we define $n$ time 
intervals and time window sizes $W$ to reflect a fading memory process $\left(F_{a}\right)$, which reduces a long past information of the environment. The procedures of DEFN consist of seven steps.

1) Measure a GBBA of EFN: We measure a GBBA of EFN using evidential operations at $t$ initially.

$1^{\text {st }}$ Prediction is equal to the $1^{\text {st }}$ measurement at $S_{1}$.

2) Update the Weight of a Sensor: We calculate individual difference between two time-indexed states using the proposed normalized weighting technique. We then apply the updated weight into each sensor from 2nd time-indexed state to obtain the GPT of information.

3) Prediction and Evolution: We calculate prediction from previous GBBA and model of evolution using the disjunctive combination rule that does not allow to assigning more belief to hypothesis than does the previous GBBA. The GBBA of EFN at time stamp $t+1$ will be affected by prediction.

4) Learning: We fuse prediction and measurement at $t+1$ using the conjunctive combination rule to make a new GBBA. Within a learning step, if a conflict value $\left(C_{F}\right)$ is greater than $T$, a new GBBA is adapted. Whereas, the previous learned GBBA is adapted continuously as a new GBBA.

5) Fading Memory Process (Fa): We apply $F a$ with the defined time window size $(W)$ to reduce the affection of a long past information. After $F a$ is performed, the GBBA of prediction at $t+w$ is equal to the GBBA of measurement at $t+w$. The previous GBBA of prediction at $t+w-1$ is ignored at $t+w$.

6) Update and Decision Making: We calculate each GPT per time-indexed state by applying updated rule then calculate differentiation $\left(D_{F}\right)$ of two consecutive time-indexed states. Based on the mean of $D_{F}$ and the pre-defined value for $\delta$ and $\gamma$, we can make a decision: No errors, False alarm, or Emergency.

7) Comparison the GPT level: Finally, we compare the GPT level. If the GPT level is over Te, which represents the emergency situation, for defined continuous time-indexed states, we make a decision about the situation of the environment as an emergency.

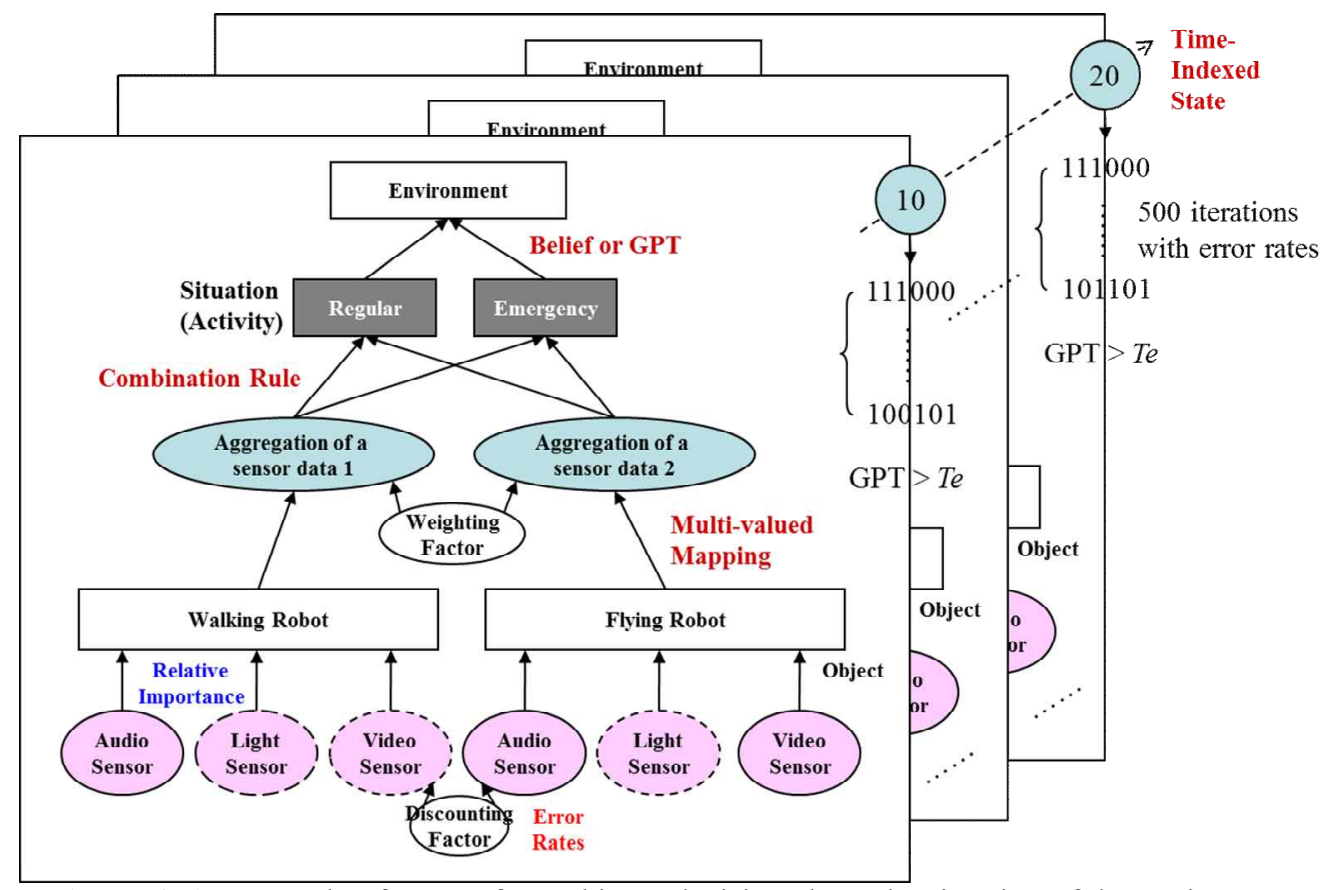

Figure 4. An example of DEFN for making a decision about the situation of the environment

\section{A Case Study}

\subsection{Applied Scenario and Assumptions}

We face many ambiguous situations of environment in intelligent multi-robot system applications. Particularly, we suppose that the situation (i.e., "Regular" (R) or "Emergency" (E)) of the environment 
can happen. In order to check dynamic emergency and to make a decision about the situation, six types of a sensor attached on the multi-robot are randomly activated during some time intervals. As six types of a sensor attached on the robot, walk-audio (wa), walk-light (wl), walk-video (wv), flying-audio (fa), flying-light (fl), and flying-video (fv) sensors are used in space and terrain. As shown in Figure 4, we perform an evidential fusion process with a $95 \%$ confidence interval for 500 iterations for making a simulation. Assuming that a discounting factor of each sensor is different. Table 1 shows an initial relative weight of each sensor using a scale representing method. Initially, a discounting factor and a relative weight of each sensor are fixed so as to calculate the initial GBBA of EFN. Moreover, we use paired observations [17] that construct a confidence interval for the difference in order to compare our method with DBNs. The analysis of paired observations deals with two processes as one process of $n$ pairs. For each pair, the difference in performance can be computed. Then, if the confidence interval includes zero, two fusion processes are not significantly different.

\section{Comparison and Analysis}

\subsection{GPT Levels of DSmT and DBNs}

We compare the GPT levels of two cases: 1) DEFN and 2) DBNs. For calculating the "Emergency (E)" situation of the environment within the applied scenario, we apply two methods: 1) weighting factors and 2) discounting factors into two fusion processes, respectively. In particular, we utilize the paired observation method with different error rates $(r)$ (i.e., $0 \%, 1 \%, 5 \%, 10 \%, 20 \%$ and $50 \%$ ) so as to compare the two fusion processes. The GPT level of each case is calculated within DEFN as the default value of the criterion. This default value is used to compare the relation dependency of DEFN with that of DBNs [18]. The model evolution (i.e., the association or correlation of two consecutive time-indexed states) of DEFN is applied as a transition probability of DBNs in order to compare the GPT of DEFN with that of DBNs.

\subsubsection{Comparison with Weighting Factors}

First, we compare the GPT level of DEFN with that of DBNs by calculating the GPT difference with a $95 \%$ confidence interval. As shown in Table 2, we apply different weights to each sensor with $T=0$ and $W=5$. Since we can apply dynamic change of the weight into the fusion process if $T=0$. If the value of $W$ is small, the frequent $F a$ can ignore the relation dependency of consecutive time-indexed states. So we apply $W=5$. We use paired observations depending on the GPT level of DEFN when the degree of GPT level is over 0.5 cases. Because the aggregation of the degree of GPT is not over 0.5 reduces the total GPT level.

As shown in Figure 5, the GPT levels of different cases have different paired observation results. The confidence interval includes zero when we compare the case 1 and case 2 . In this case, it is impossible to distinguish which one is better than the other. The reason is that the degree of GPT is lower than 0.5 sometimes. Whereas the confidence intervals of the case 3 and 4, the case 5 and 6 and the case 7 and 8 do not have zero. In these cases, we can prove that the GPT levels of DEFN with different weights are better than those of DBNs. In addition, we can distinguish which one is better than the other with $0 \%, 1 \%, 5 \%, 10 \%$, or $20 \%$ error rate. However, it is impossible to prove anything with a $50 \%$ error rate. Because an error rate makes the wrong simulation operation then the result is nothing.

Table 2. An example of different weights for DEFN and DBNs

\begin{tabular}{|c|c|c|c|c|c|c|}
\hline Case & $w a$ & $w l$ & $w v$ & $f a$ & $f l$ & fv \\
\hline 1 (DEFN) & 0.8 & 0.1 & 0.1 & 0.3 & 0.4 & 0.3 \\
\hline 2 (DBNs) & 0.8 & 0.1 & 0.1 & 0.3 & 0.4 & 0.3 \\
\hline $3(\mathrm{DEFN})$ & 0.5 & 0.25 & 0.25 & 0.25 & 0.25 & 0.5 \\
\hline 4 (DBNs) & 0.5 & 0.25 & 0.25 & 0.25 & 0.25 & 0.5 \\
\hline $5(\mathrm{DEFN})$ & 0.3 & 0.2 & 0.5 & 0.17 & 0.33 & 0.5 \\
\hline $6(\mathrm{DBNs})$ & 0.3 & 0.2 & 0.5 & 0.17 & 0.33 & 0.5 \\
\hline 7 (DEFN) & 0.2 & 0.3 & 0.5 & 0.3 & 0.3 & 0.4 \\
\hline 8 (DBNs) & 0.2 & 0.3 & 0.5 & 0.3 & 0.3 & 0.4 \\
\hline
\end{tabular}




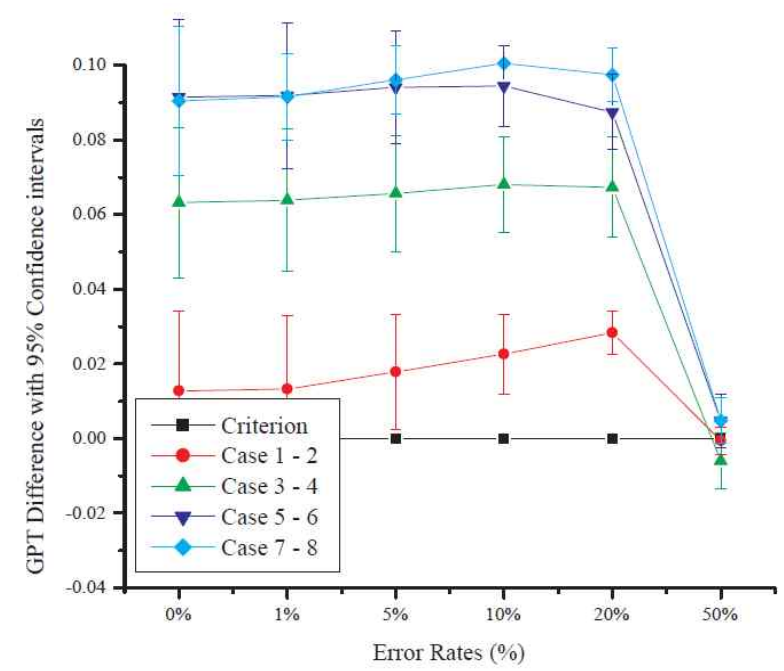

Figure 5. Comparison GPT levels of DEFN with those of DBNs based on different weighting factors

Table 3. An example of different discounting factors $D$

\begin{tabular}{c|c|c|c|c|c|c}
\hline Case No. & $\boldsymbol{w a}$ & $\boldsymbol{w l}$ & $\boldsymbol{w v}$ & $\boldsymbol{f a}$ & $\boldsymbol{f l}$ & $\boldsymbol{f} \boldsymbol{v}$ \\
\hline Case 1 & $20 \%$ & $10 \%$ & $0 \%$ & $20 \%$ & $10 \%$ & $0 \%$ \\
Case 2 & $20 \%$ & $10 \%$ & $1 \%$ & $20 \%$ & $10 \%$ & $1 \%$ \\
Case 3 & $20 \%$ & $10 \%$ & $2 \%$ & $20 \%$ & $10 \%$ & $2 \%$ \\
Case 4 & $20 \%$ & $10 \%$ & $5 \%$ & $20 \%$ & $10 \%$ & $5 \%$ \\
Case 5 & $20 \%$ & $10 \%$ & $10 \%$ & $20 \%$ & $10 \%$ & $10 \%$ \\
Case 6 & $20 \%$ & $10 \%$ & $20 \%$ & $20 \%$ & $10 \%$ & $20 \%$ \\
Case 7 & $20 \%$ & $10 \%$ & $50 \%$ & $20 \%$ & $10 \%$ & $50 \%$ \\
\hline
\end{tabular}

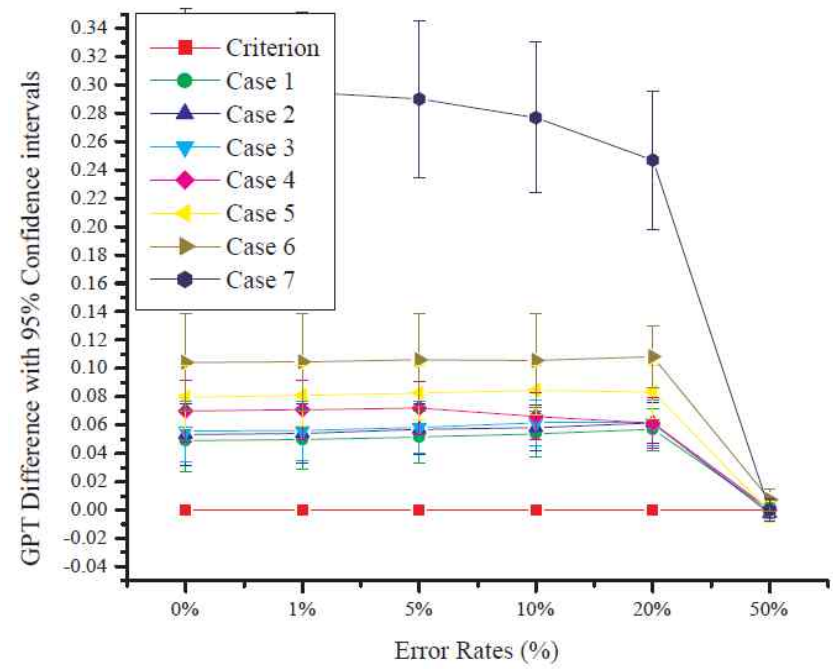

Figure 6. Comparison GPT levels of DEFN with those of DBNs based on different discounting factors

\subsubsection{Comparison with Discounting Factors}

Second, we compare the GPT level of DEFN with that of DBNs with different discounting factors by applying different discounting factors into each sensor as shown in Table 3. In particular, we compare the GPT level of the two processes depending on different D on " $w v$ " and "fv", because we assume that sensed video information more reliable than the other information in space and terrain. The two cases show different degrees of GPT levels as shown in Figure 6. The GPT levels of DBNs are lower than those of the DEFN except for the $50 \%$ error rate of " $w v$ " and " $f v$ " case when we utilize 
paired observations for all cases in Table 3. Based on the result of Figure 6, the confidence intervals do not include zero except for the error rate is $50 \%$ case. Our approach with different discounting factors gets a higher confidence level of information than the fusion process based on DBNs.

\subsection{Decision Making}

To check the emergency situation of the environment, $2^{6}$ cases that depends on activated sensors happen randomly during 50 time intervals as shown in Figure 7. The level change of the state of a sensor represents temporal changes in sensory information in order to find a false alarm. To make a simulation, we apply different simulation error rates (e.g., $0 \%, 20 \%$ and $50 \%$ ) into the evidential fusion process with a $95 \%$ confidence interval for 500 iterations.

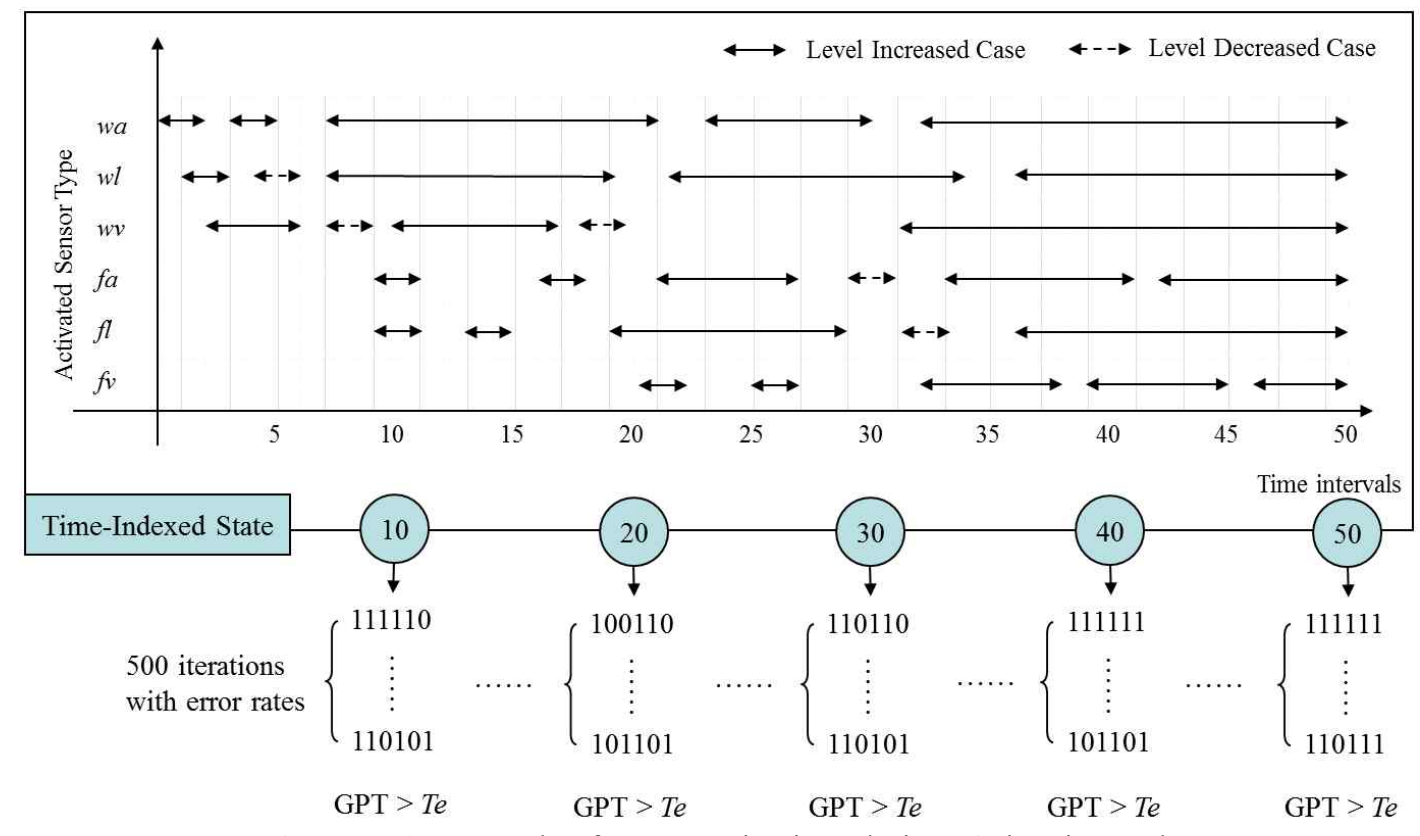

Figure 7. An example of sensor activations during 50 time intervals

\subsubsection{Finding an Optimal Time Window Sizes $W$}

In order to make a correct decision about the "emergency", first we have to define the time window size $(W)$ that supports a fading memory process $(F a)$. We assume that $W$ is 5 because the longer $W$ can have a difficult to catch a false alarm or an emergency progress. For instance, if we apply the longer time window size such as $W=35$ or $W=15$, the mean of the differentiation $\left(D_{F}\right)$ between two consecutive time-indexed states has no variations as shown in Figure 8 . It is difficult to find a false alarm or an emergency progress if we utilize the longer $W$. In addition, we are able to distinguish a false alarm from new sensor activations if we check the mean of differentiation frequently. However, the shorter $W$ also has problem that there is no relation dependency between two consecutive time-indexed state then lead to decrease the GPT level of information. A trade-off exists between the increased GPT level of the DEFN approach and the mean of differentiation $\left(D_{M}\right)$. Thus, we assume that $\delta$ is equal to 0.05 and $\gamma$ is equal to 0.08 in order to make a correct decision. We utilize the middle value of $W$ as an optimal time window size (e.g., $W=5)$

\subsubsection{Decision Making for Emergency Analysis}

Finally, we can infer the situation of the environment by using the mean of the $D F$ (i.e., $D_{M}$ ) and pre-defined rule of a decision. It then can make a decision that the inferred situation has wrong 
information or not. For example, we assume that the pre-defined threshold (i.e., $T e$ ) for an emergency situation of the environment is equal to 0.7 . If the degree of GPT is over 0.7 for predefined continuous time-indexed states, we estimate that the situation is an emergency. If that is not over 0.7 for at any time-indexed states and is not match with the pattern of emergency progress, we estimate that one of sensors or some sensors have reading errors then lead to wrong decision making.

According to Figure 9, the GPT level of DEFN is higher than that of DBNs with a $0 \%$ and a $20 \%$ error rate when the degree of GPT is over 0.5 (e.g., time intervals from $17^{\text {th }}$ ). However, the GPT level of DEFN and that of DBNs is difficult to distinguish with a $50 \%$ error rate. In addition, we catch a false alarm at two points: 1) between $20^{\text {th }}$ and $25^{\text {th }}$ time intervals and 2) between $30^{\text {th }}$ and $35^{\text {th }}$ time intervals, respectively. Then, we are able to approximately estimate that the emergency situation of the environment starts from $25^{\text {th }}$ time interval. This is helpful to make a decision about the situation of environment in intelligent multi-robot system applications.

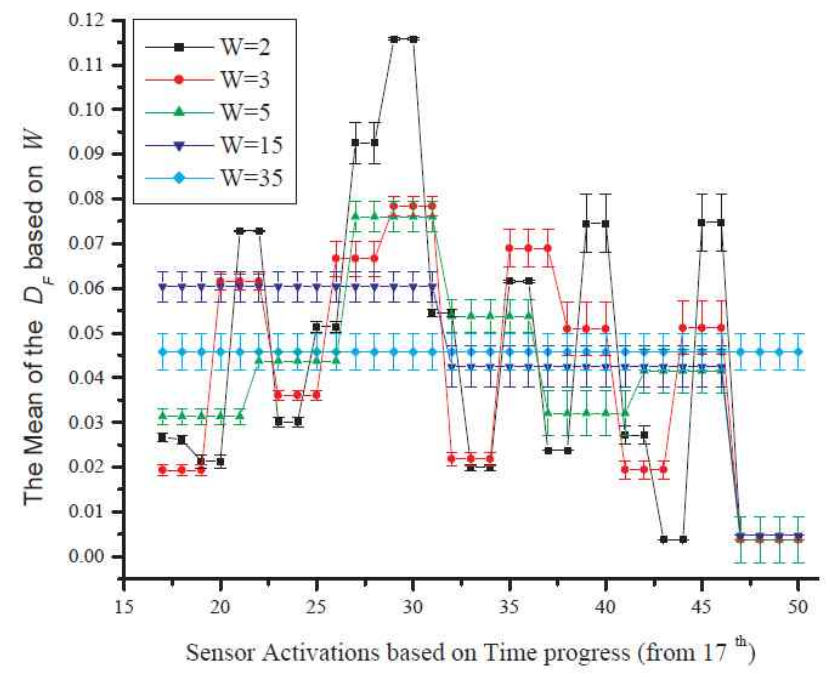

Figure 8. Comparison the mean of the differentiation with different time window sizes $(W)$

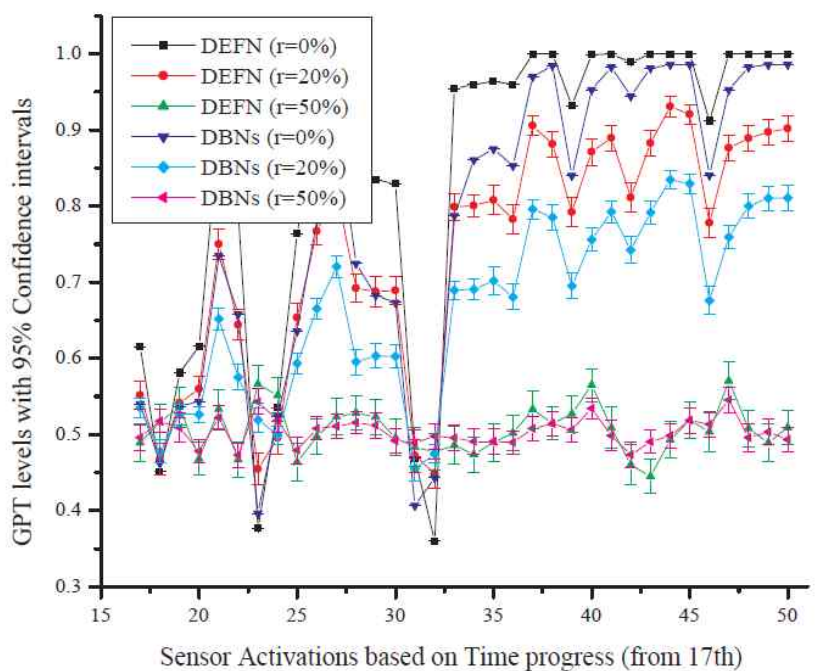

Figure 9. GPT levels of DEFN and DBNs depending on the applied scenario

\section{Related Work}

We need reasoning context models that can adapt the situation definitions based on discovered changes with changing environments and changing user needs [19]. However, both the physical world 
itself and our measurements of it are prone to uncertainty. Different types of entities in smart environments must be able to reason about uncertainty. To solve these problems, a number of mechanisms have been proposed in the literature for reasoning on uncertainty. There are two main purposes for reasoning on uncertainty: 1) improving the quality of information and 2) inferring new kinds of information. Reasoning to improve the quality of information typically takes the form of multi-sensor fusion where data from different sensors are used to increase confidence, resolution or any other quality metrics. Reasoning to infer new information typically takes the form of deducing higher level contexts (e.g., activity of a user or a robot) or situations from lower-level information, because we cannot directly sense the higher-level information. These contexts may be associated with a certain level of uncertainty depending on both the accuracy of the sensed information and precision of the deduction process [20], [21]. Therefore, we introduce some reasoning approaches such as Fuzzy logic, Probabilistic logic, Bayesian Networks (BNs), Hidden Markov Models (HMMs), Kalman Filtering Models (KFMs), Dynamic Bayesian Networks (DBNs) and Dempster-Shafer Theory (DST) of the evidence in order to compare them with our reasoning approach.

\subsection{Puzzy Logic, Probabilistic Logic and BNs}

In fuzzy logic, a degree of membership represented by a pair $(A: m)$ where $A$ is a set and $m$ is a possibility distribution in real unit interval $[0,1]$ is used to show an imprecise notion such as confidence values [22], [23]. The elements of two or more fuzzy sets can be combined to create a new fuzzy set with its own membership function then it is used for reasoning models which need more than the probabilistic theory with uncertainty. For example, fuzzy logic is used to capture a clinical uncertainty in medical data of pervasive computing applications in [24]. In addition, fuzzy logic is well suited for describing subject contexts by resolving conflicts between different actuator's operations [25]. We assume that the sensors attached on multi-robot system are operated based on the fuzzy logic of the selected sensors.

Probabilistic logic and Bayesian networks (BNs) can be used for improving the quality of information through multi-sensor fusion as well as for deriving the higher-level probabilistic contexts. They also can be used for resolving conflicts between information obtained from different sources. According to [26], [27], the probabilistic logic is used for encoding access control policies and the BNs is used for combining uncertain information from a large number of sources and deducing higher-level contexts. However, these rules can not represent the ignorance [28], which manages the degree of uncertainty, caused by the lack of information.

\subsection{HMMs, KFMs and DBNs}

In order to dealing with unpredictable temporal changes in obtained sensory information, Hidden Markov Models (HMMs) [29], [30], [31], Kalman Filtering Models (KFMs) [32], [33] or Dynamic Bayesian Networks (DBNs) [6], [11], [18] are used as fusion techniques. In terms of probabilistic networks, HMMs represent stochastic sequences as Markov chains. This model is used for location prediction by using a hierarchical Markov model that can learn and infer a user's daily movements [34]. KFMs represent the state of the system refers to a set of variables that describe the inherent properties of the system at a specific instant of time. This is a useful technique for estimating, or updating the previous estimate of, a system's state by using indirect measurements of the state variables and using the covariance information of both state variables and indirect measurements [35].

However, DBNs, which were proposed as a generalization of HMMs and KFMs, have some distinct features. DBNs allow much more general graph structures compared with HMMs or KFMs. DBNs represent the hidden state in terms of a set of random variable compared with HMMs, which represent the state space with a single random variable. DBNs allow general hybrid and nonlinear conditional probability densities (CPDs) compared with KFMs, which require all CPDs to be linearGaussian. This is a useful feature to manage the causality between random variables as well as time series data. For instance, a high level user behavior is inferred from low level sensor data by adding knowledge of real-world constraints to user location data in [36]. A variant of DBNs is used in an unsupervised way in order to predict transport routes based on GPS data. By adding constraints on the routes that could be learned by the training algorithm, the prediction accuracy was significantly improved. 
DBNs are made up of the interconnected two time-indexed states of a static Bayesian Network $(\mathrm{BN})$ and the transition of a static $\mathrm{BN}$ between two consecutive time $t$ and $t+1$ satisfies the Markov property [37]. In this work, we use Markov property, which is similar to DBNs, in order to represent temporal and state links between two consecutive time-indexed states of DEFN then compare it with the original process of DBNs.

\subsection{Dempster-Shafer Theory (DST)}

DST is a mathematical theory of the evidence based on belief and plausible reasoning, which is used to combine separate pieces of information in order to calculate the probability of the event. It is often used method of sensor fusion to deal with uncertainty associated with context reasoning by combining the independent observations of multiple sensors (e.g., the user's activity monitoring in smart home) [38], [39]. However, the DST has limitations and weaknesses. In particular, the Dempster's combination rule has limitations. The results of the combination have low confidences when a conflict becomes important between sources. Thus, we use the Dezert-Smarandache Theory (DSmT), which is an extended DST, as a reasoning method. No one applies the DSmT into pervasive computing area or smart environmental applications such as multi-agent system or multi-robot system. Our research attempts the DSmT into the pervasive computing applications and intelligent multi-robot system in order to reduce the conflicting mass in uncertainty level of information and improve the confidence (i.e., GPT) level of information in emergency situation of the environment.

\section{Conclusion}

Until now, we proposed a reasoning method under uncertainty based on Dynamic Evidential Fusion Network (DEFN) in smart environments in order to support both consistency verification of the model and reasoning techniques in multi-robot system. The proposed reasoning technique improved the quality of information and inferred new kinds of information. The proposed DEFN dealt with dynamic metrics such as preference, temporal consistency and relation-dependency of the consecutive timeindexed state using the autonomous learning method such as normalized weighting technique and Temporal Belief Filtering (TBF) then improved the confidence level of information compared to the fusion process based on DBNs. In particular, we compared the confidence (i.e., GPT) levels of the two fusion processes with different weighting factors and discounting factors using paired observations in order to show the improvement of our approach. We then showed an example of a false alarm that helps to decision making more correctly.

We will continuous work on autonomous learning process with self-configuring, self-healing, selfoptimizing, and self-protecting in order to adapt the various situation of the environment into the multirobot system without errors. This research directions will be able to make Cyber Physical System (CPS) in the real world by improving dependable and reliable information of the system..

\section{Acknowledgements}

This work was supported by the Sun Moon University Research Grant of 2012.

\section{References}

[1] Maria Valera Espina, Raphael Grech, Deon De Jager, Paolo Remagnino, Luca Locchi, Luca Marchetti, Daniele Nardi, Dorothy Monekosso, Mircea Nicolescu, and Christopher King, "Multirobot teams for environmental monitoring," Springer, Studies in Computational Intelligence, vol. 336, pp. 183-210, 2011.

[2] L. Grajales and I. V. Nicolaescu, "Wearable multi-sensor heart rate monitor," in Proc. of the Intl. Workshop on Wearable and Implantable Body Sensor Networks (BSN'06), pp. 31-36, 2006.

[3] T. Said, S. Ghoniemy, G. ISmail, and I. A. EL-Ghafar, "Multi-object color tracking for multirobot systems enhancement," in the 13th Intl. Conf. on Aerospace Science and Aviation Technology, pp. 1-11. 2009. 
[4] Jean Dezert and Florentin Smarandache, Advances and Applications of DSmT for Information Fusion, Vol. 3. Rehoboth: American Research Press, USA, 2009.

[5] Florentin Smarandache and Jean Dezert, "Information fusion based on new proportional conflict redistribution rules," in Proc. of the 8th Intl. Conf. on Information Fusion, pp. 907-914, 2005.

[6] Jean Dezert, Florentin Smarandache, and Milan Daniel, "The generalized pignistic transformation," in Proc. of the 7th Intl. Conf. on Information Fusion, pp. 384-391, 2004.

[7] Hyun Lee, Jae Sung Choi, and Ramez Elmasri, "Sensor data fusion using DSM theory for activity recognition under uncertainty in home-based care," in Proc. of the 23th IEEE Intl. Conf. on Advanced Information Networking and Applications (AINA’09), pp. 517-524, 2009.

[8] Galina L. Rogova and Vincent Nimier, "Reliability in information fusion: literature survey," in Proc. of the 7th Intl. Conf. on Information fusion, pp. 1158-1165, 2004.

[9] Hyun Lee, Jae Sung Choi, and Ramez Elmasri, "A classification and modeling of the quality of contextual information in smart spaces," in Proc. of the 6th IEEE Intl. PerCom Workshop on Context Modeling and Reasoning (CoMoRea'09), pp. 1-5, 2009.

[10] George Valiris, Panagiotis Chytas, and Michael Glykas, "Making decisions using the balanced scorecard and the simple multi-attribute rating technique," Performance Measurement and Metrics, vol. 6, no. 3, pp. 159-171, 2005.

[11] Kevin Patrick Murphy, Dynamic Bayesian Networks: Representation, Inference and Learning. Univ. of California, Berkeley: Ph. D. Dissertation, USA, 2002.

[12] Jean Dezert and Florentin Smarandache, Advances and Applications of DSmT for Information Fusion, Vol. 1. Rehoboth: American Research Press, USA, 2004.

[13] Glenn Shafer, A Mathematical Theory of Evidence. Princeton, New Jersey: Princeton Univ. Press, USA, 1976.

[14] E. Ramasso, M. Rombaut, and D. Pellerin, "A temporal belief filter improving human action recognition in videos," in Proc. of the IEEE Intl. Conf. on Acoustics, Speech, and Signal Processing (ICASSP'06), pp. 141-144, 2006.

[15] Philippe Smets, "Data fusion in the transferable belief model," in Proc. of the 3rd Intl. Conf. on Information Fusion, pp. 21-33, 2000.

[16] D. von Winterfeldt and W. Edwards, Decision Analysis and Behavioral Research. University Press, Cambridge, UK: Cambridge, UK. 1986.

[17] Raj Jain, "The Art of Computer Systems Performance Analysis Techniques for Experimental Design, Measurement, Simulation, and Modeling", Digital Equipment Corporation, Littleton, Massachusetts: John Wiley \& Sons, Inc., USA, 1991.

[18] Yongmian Zhang and Qiang Ji, "Active and dynamic information fusion for mult-isensor systems with dynamic Bayesian Networks,” IEEE Trans. Syst., Man, Cybern. B, vol. 36, no. 2, pp. 467472, 2006.

[19] Prem Jayaraman, Arkady Zaslavsky, and Jerker Delsing, "Dynamic situation modeling and reasoning under uncertainty," in Proc. of the Intl. Conf. on Pervasive Services (ICPS'09), pp. 113-122, 2009

[20] Claudio Bettini, Oliver Brdiczka, Karen Henricksen, Jadwiga Indulska, Daniela Nicklas, Anand Ranganathan, and Daniele Riboni, "A survey of context modeling and reasoning techniques," Pervasive and Mobile Computing, vol. 6, no. 2, pp. 161-180, 2009.

[21] Hyun Lee, Byung Yong Lee, Kyung Seo Park, and Ramez Elmasri, "Fusion techniques for reliable information: A survey," JDCTA: International Journal of Digital Content Technology and its Applications, vol. 4, no. 2, pp. 74-88, 2010.

[22] L. A. Zadeh, "Fuzzy sets as a basis for a theory of possibility," Fuzzy Sets and Systems, vol. 100, no. 1, pp. 9-34, 1999.

[23] Enrica Chiappero Martinetti, "Capability approach and fuzzy set theory: description, aggregation and inference issues," Fuzzy set approach to multidimensional proverty measurement, vol. 3, pp. 93-113, 2006.

[24] Sheetal Agarwal, Anupam Joshi, Tim Finin, Yelena Yesha, and Tim Ganous, "A pervasive computing system for the operating room of the future," Mobile Networks and Applications, vol. 12, no. 2, pp. 215-228, 2007.

[25] Hyun Lee, Jae Sung Choi, and Ramez Elmasri, "A conflict resolution architecture for the comfort of occupants in intelligent office," in Proc. of the 4th IET Intl. Conf. on Intelligent Environments (IE’08), pp. 1-8, 2008. 
[26] A. Ranganathan, J. Al-Muhtadi, and R. H. Campbell, "Reasoning about uncertain contexts in pervasive computing environments," IEEE Pervasive Computing, vol. 3, no. 2, pp. 62-70, 2004.

[27] Tao Gu, Hung Keng Pung, and Da. Qing Zhang, “A Bayesian approach for dealing with uncertain contexts," in Proc. of the 2nd Intl. Conf. on Pervasive Computing, Austrian Computer Society, pp. 205-210, 2004.

[28] Simon Maskell, "A Bayesian approach to fusing uncertain, imprecise and conflicting information," Information Fusion, vol. 9, no. 2, pp. 259-277, 2008.

[29] Waltenegus Dargie, "The role of probabilistic schemes in multisensory context-awareness," in Proc. of the 5th IEEE Intl. Conf. on Pervasive Computing and Communications Workshops, pp. 27-32, 2007.

[30] Nuria Oliver and Eric Horvitz, "A comparison of HMMs and dynamic Bayesian networks for recognizing office activities," LNCS, User Modeling, vol. 3538, pp. 199-209, 2005.

[31] C. Soyer, H. I. Bozma, and Y. Isterfanopulos, "Attentional sequence-based recognition: Markovian and evidential reasoning," IEEE Trans. Syst., Man, Cybern. B, vol. 33, no. 6, pp. 937950, 2003.

[32] Peter. S. Maybeck, Stochastic Models, Estimation, and Control. New York: Academic, USA, 1979.

[33] Greg Welch and Gary Bishop, An Introduction to the Kalman Filter. TR 95-041: UNC-Chapel Hill, USA, 2006.

[34] Lin Liao, Dieter Fox, and Henry Kautz, "Learning and inferring transportation routines," Artificial Intelligence, vol. 171, no. 5-6, pp. 311-331, 2007.

[35] R. Olfati-Saber, "Distributed Kalman filtering for sensor networks," in Proc. of the 46th IEEE Intl. Conf. on Decision and Control, pp. 5492-5498, 2007.

[36] Donald J. Patterson, Lin Liao, Dieter Fox, and Henry Kautz, "Inferring high-level behavior from low level sensors," in Proc. of the 5th Intl. Conf. on Ubiquitous Computing (UbiComp'03), pp. 73-89, 2003.

[37] Padhraic Smyth, "Belief networks, hidden Markov model, and Markov random field: A unifying view," Pattern Recognition letters, vol. 18, pp. 1261-1268, 1997.

[38] Huadong $\mathrm{Wu}$, Sensor data fusion for context-aware computing using Dempster-Shafer theory. CMU: Ph. D. Dissertation, USA, 2004.

[39] Xin Hong, Chris Nugent, Maurice Mulvenna, Sally McClean, Bryan Scotney, and Steven Devlin, "Evidential fusion of sensor data for activity recognition in smart homes," Pervasive and Mobile Computing, vol. 5, no. 3, pp. 236-252, 2009.

[40] Yang Yuequan, Jin Lu, Cao Zhiqiang, Tang Hongru, and Xia Yang and Ni Chunbo, "A survey of reinforcement learning research and its application for multi-robot systems", $31^{\text {st }}$ Chinese Control Conference, pp. 3068-3074, 2012.

[41] T. Yasuda, K. Kage, and K. Ohkura, "Robust reinforcement learning technique with bigeminal representation of continuous state space for multi-robot systems", SICE Annual Conference, pp. 1552-1557, 2012.

[42] Jiansheng Peng and Xing Li, "The Research of Improving the Fish Swarm Algorithm for Robot Path Planning", IJACT: International Journal of Advancements in Computing Technology, vol. 5, no. 1 , pp. $187 \sim 195,2013$. 